\title{
Ballistic Hall micromagnetometry
}

\author{
A. K. Geim \\ Research Institute for Materials, University of Nijmegen, 6525 ED Nijmegen, The Netherlands \\ S. V. Dubonos \\ Institute for Microelectronics Technology, Russian Academy of Sciences, 142432 Chernogolovka, Russia \\ J. G. S. Lok, I. V. Grigorieva, and J. C. Maan \\ Research Institute for Materials, University of Nijmegen, 6525 ED Nijmegen, The Netherlands \\ L. Theil Hansen and P. E. Lindelof \\ University of Copenhagen, Niels Bohr Institute, Universitetsparken 5, DK-2100 Copenhagen, Denmark
}

(Received 27 May 1997; accepted for publication 22 August 1997)

We report a magnetization measurement technique which allows quantitative studies of thermodynamic properties of individual submicron superconducting and ferromagnetic particles.

(C) 1997 American Institute of Physics. [S0003-6951(97)02042-1]

Hall and magnetoresistive probes have been employed for studying magnetic properties of various materials for several decades. ${ }^{1}$ Most recently, advances in microtechnology have allowed fabrication of such sensors of micron size and they were successfully applied for time- and space-resolved detection of individual vortices in superconductors. ${ }^{2-4}$ All previous studies have focused on the properties of macroscopic samples. The only exception is Ref. 5 in which Hall probes were combined with microfabricated arrays of ferromagnetic particles. In the present paper, we report on a further development in Hall probe techniques by employing submicron ballistic Hall probes for studying individual submicron samples. The use of Hall probes in the regime of ballistic electron transport and samples of size smaller than the probe size makes a qualitative difference, allowing us to make a link between the detected signal and the sample magnetization. In brief, our technique can be described as a solidstate analog of the Lorenz electron microscopy, where an electron beam passes in a close vicinity of a small magnetized object and the deviations of the beam due to a stray magnetic field are detected. ${ }^{6}$ For the case of the ballistic Hall probe, instead of an electron beam we use a ballistic electron flow confined within a narrow metal wire. The Hall leads that are symmetrically attached to the central wire serve as a sensitive detector of the smallest deviations in the direction of the electron "beam."

Figure 1 shows an SEM micrograph of one of our working devices. It is a multiterminal wire (a set of five Hall crosses) fabricated from a GaAs/GaAlAs heterostructure with a high-mobility two-dimensional electron gas (2DEG) embedded at $60 \mathrm{~nm}$ below the surface. To make such devices, we have used two stages of photolithography to define contact pads and conventional AuGeNi contacts to the 2DEG and leave the 2 DEG only within a central area of about 100 $\times 100 \mu \mathrm{m}^{2}$ between them. This area is subsequently used to make in the 2DEG the wire pattern shown in Fig. 1 with the help of electron-beam lithography having the resolution of about $0.1 \mu \mathrm{m}$. Using the second round of electron-beam lithography, we expose the resist to make openings of various sizes in the central, sensitive area of the Hall crosses (this procedure requires high accuracy of the pattern alignment). The final step is evaporation of the required material and liftoff. In Fig. 1 the samples are four $\mathrm{Al}$ disks of the diameter $d$ from 0.2 to $1 \mu \mathrm{m}$ (thickness $0.15 \mu \mathrm{m}$ ) and the last Hall probe is left empty to serve as a reference. The presence of a diamagnetic or ferromagnetic sample results in either an expulsion or, conversely, in an increased value of the magnetic field inside the cross junction, which leads to a decrease or an increase in the Hall voltage, respectively. The detected changes can be compared with the Hall signal at the reference cross which measures the applied magnetic field $H$.

We have used Hall probes with conductive channel widths $W$ down to $0.2 \mu \mathrm{m}$, but for the purpose of studying several submicron samples in the same run and finding a size dependence in their magnetic response, Hall probes with $W$ $\approx 1 \mu \mathrm{m}$ are found to be most convenient. One of the reasons for using a $2 \mathrm{DEG}$ is its large Hall coefficient (1/ne), due to a relatively low concentration of $2 \mathrm{D}$ electrons $(n \approx 3$ $\times 10^{11} \mathrm{~cm}^{-2}$ ). We note also that the top layer of the heterostructure provides a perfect electrical insulation between the studied samples and the $2 \mathrm{DEG}$, so that any possible variation in the sample conductance does not directly influence the 2DEG. However, the most important reason for using a 2DEG in our studies is its high mobility $\left(3 \times 10^{5}\right.$ $\mathrm{cm}^{2} \mathrm{~V}^{-1} \mathrm{~s}^{-1}$, in our case), such that electrons move ballistically inside the cross junction and experience scattering at the boundaries only. Unlike diffusive transport, ballistic transport allows a straightforward quantitative description of

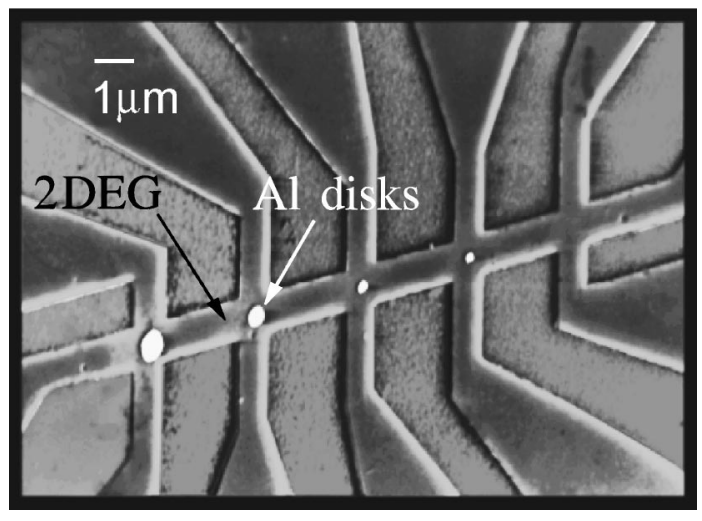

FIG. 1. SEM micrograph of our ballistic Hall micro-magnetometers with several mesoscopic samples in the sensitive area of the probes. 
the detected Hall signal. In the former case, the Hall response depends on the details of the magnetic field distribution $B(x, y)$ inside and near the cross junction and, therefore, only rapid or oscillatory changes in magnetization can be reliably identified. In the ballistic regime and in low magnetic fields (such that the size of a cyclotron orbit is larger than $W$ ), the following uncomplicated relation appears to be valid:

$$
R_{x y}=\alpha\langle B\rangle / n e ;
$$

i.e., the Hall effect simply measures the average magnetic field in the cross junction, $\langle B\rangle$. This result has been obtained analytically for a square junction with sharp corners ${ }^{7}$ and extended numerically for square junctions with rounded corners and rectangular junctions. ${ }^{8}$ The average is taken over a characteristic area $S$ of the cross junction [for a square junction with rounded corners, $S \approx(W+1.4 r)^{2}$, where $r$ is the radius of the corners ${ }^{8}$ ]. The Hall coefficient $\alpha$ generally differs from unity due to collimation effects for ballistic electrons, and it is typically 1.2 in our devices with slightly rounded corners. The average magnetic field $\langle B\rangle$ is $\int B d x d y / S=\Phi / S$; i.e., the measured signal is determined by the flux $\Phi$ through the characteristic area of the Hall cross. One can easily see that a ballistic Hall probe works exactly as a micro-fluxmeter, similar to, e.g., SQUIDs, but with a tiny detection loop. For our smallest devices, $S$ is only $0.1 \mu \mathrm{m}^{2}$. Knowing the average magnetic field $\langle B\rangle$ $=n e R_{x y} / \alpha$, we can define the area magnetization as

$$
4 \pi M=\langle B\rangle-H \text {. }
$$

Like with any other fluxmeter, the magnetization signal depends on the filling factor, i.e., on the ratio between the sample and junction size. For our disk-shaped particles, $M$ is found to vary approximately as $d^{2} / W^{3}$, in agreement with straightforward calculations.

Figure 2 shows examples of the magnetization curves measured on some of our superconducting samples and the smallest ferromagnetic particles that we could fabricate by electron-beam lithography. In order to demonstrate that our technique works even at nitrogen temperatures, where the 2DEG transport is still ballistic, we present the magnetization curve for a $100 \mathrm{~nm}$ ferromagnetic particle at $60 \mathrm{~K}$. Comparison between the amplitudes of the magnetization signals for the ferromagnetic and superconducting particles in Fig. 2 shows clearly that much smaller ferromagnetic particles can be investigated even when using the relatively large $1 \mu \mathrm{m}$ Hall probes.

In a constant magnetic field, the sensitivity of our measurements was limited by Johnson noise, in agreement with Ref. 3. At nitrogen temperatures, our Hall probes with $W$ $=1 \mu \mathrm{m}$ have the series resistance of about $4 \Omega$ and can sustain currents of up to $100 \mu \mathrm{A}$, thus enabling the field resolution $\delta B$ of about $10^{-4} \mathrm{Ghz}^{-1 / 2}$. $^{3}$ This yields an impressive flux resolution $\delta \Phi=\delta B S \approx 10^{-5} \phi_{0}$ at $77 \mathrm{~K}$ for our nonoptimized design. In terms of the magnetic moment, this sensitivity allows us to detect changes $\delta M \approx 10^{3} \mu_{B}$. The flux resolution slightly improves for smaller probes with $W$ $\approx 0.2 \mu \mathrm{m}$. However, in contrast to what one might expect from the known temperature dependence of Johnson noise, we did not observe any substantial increase in the sensitivity
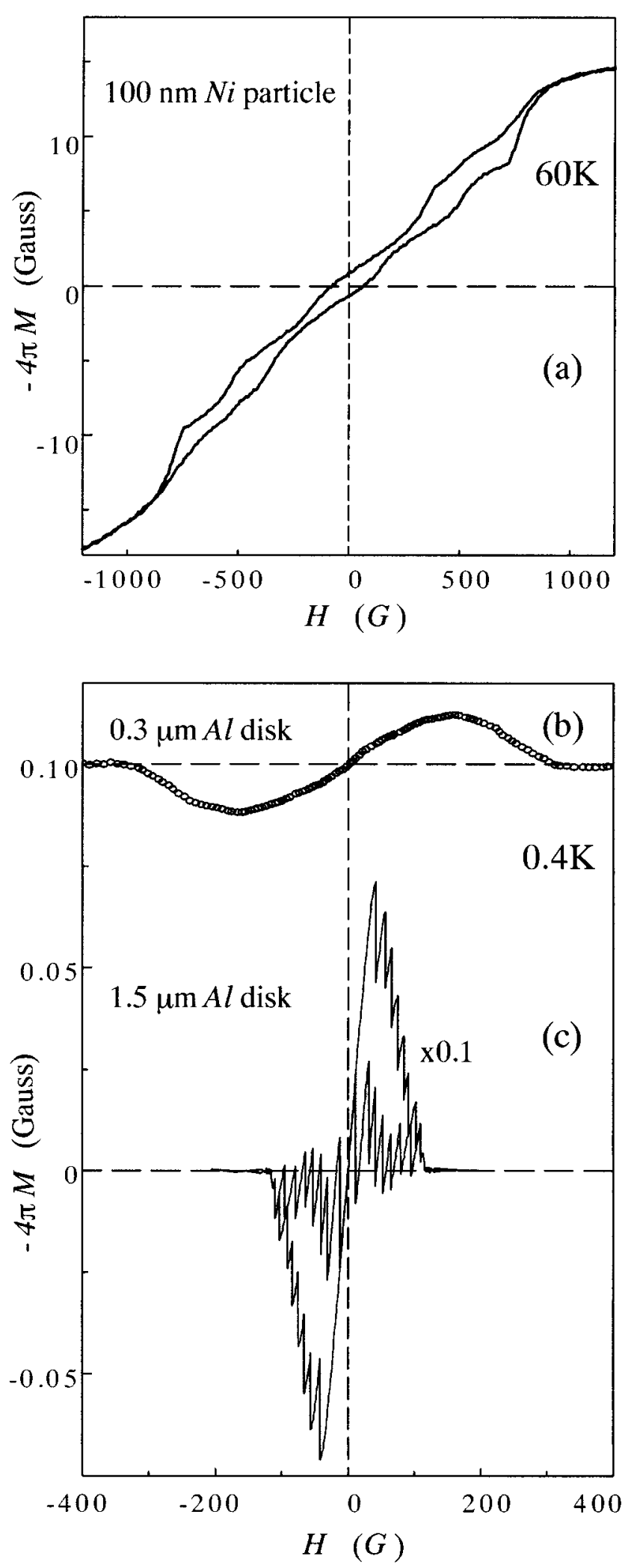

FIG. 2. Magnetization of individual ferromagnetic (a) and superconducting (b) and (c) particles. (a) Ni disk with both diameter and height $\approx 100 \mathrm{~nm}$; (b) and (c) Al disks of approximately the same height and the diameters 0.3 and $1.5 \mu \mathrm{m}$, respectively.

of the technique with decreasing temperature down to $0.3 \mathrm{~K}$. At low temperatures, the flux resolution even decreases due to the fact that the probes appear to be able to sustain only smaller currents of about $10 \mu \mathrm{A}$ per $1 \mu \mathrm{m}$ width. We note that such currents heat up the $2 \mathrm{DEG}$ to electron temperatures 
above $20 \mathrm{~K}$, but this does not cause any noticeable changes in the sample temperature. The latter could be detected as a shift in the values of a critical magnetic field in a superconducting sample and were found to be negligibly small even at $0.3 \mathrm{~K}$ and for samples mounted in vacuum (i.e., not immersed in a $\mathrm{He} 3$ bath).

If magnetization is measured versus the applied magnetic field as in Fig. 2, there is an additional source of error due to mesoscopic conductance fluctuations in the 2DEG at low temperatures. Even at $20 \mathrm{~K}$, the fluctuations lead to a probe-specific background in the Hall signal which can be hard to distinguish from a sample response, particularly if it is smooth. For superconducting samples, we found it convenient to measure $M$ as the difference between the Hall signals measured at the same probe just above the superconducting transition temperature of $\approx 1.2 \mathrm{~K}$ [where $\langle B\rangle=H$ ] and below it. This allows us to avoid the contribution of the nonlinear background but, in any case, the field resolution in the sweeping field regime generally decreases to $\approx 0.01$ to $0.1 \mathrm{G}$, depending on the field range.

In conclusion, we have developed a simple and reliable technique which makes it possible to study magnetization of individual samples of submicron and even nanometer size in a very wide range of temperatures. By optimizing the probe design (higher driving currents at low temperatures, smaller size and series resistance), we believe that it is feasible to reach the level of single electron spin detection.

${ }^{1}$ H. T. Coffey, Cryogenics 7, 73 (1965); R. N. Goren and M. Tinkham, J. Low Temp. Phys. 5, 465 (1971).

${ }^{2}$ D. A. Brawner, N. P. Ong, and Z. Z. Wang, Nature (London) 358, 567 (1992); A. K. Geim, I. V. Grigorieva, and S. V. Dubonos, Phys. Rev. B 46, 324 (1992); S. T. Stoddart, S. J. Bending, A. K. Geim, and M. Henini, Phys. Rev. Lett. 71, 3854 (1993); E. Zeldov, A. I. Larkin, V. B. Geshkenbein, M. Konczykowski, D. Majer, B. Khaykovich, V. M. Vinokur, and H. Shtrikman, Phys. Rev. Lett. 73, 1428 (1994).

${ }^{3}$ A. Oral and S. J. Bending, Appl. Phys. Lett. 69, 1324 (1996).

${ }^{4}$ A. M. Chang, H. D. Hallen, L. Harriot, H. F. Hess, H. L. Kao, J. Kwo, R. E. Miller, R. Wolfe, and J. van der Ziel, Appl. Phys. Lett. 61, 1974 (1992).

${ }^{5}$ A. D. Kent, S. von Molnar, S. Gider, and D. D. Awschalom, J. Appl. Phys. 76, 6656 (1994). One might also be interested in the work by B. C. Webb and S. Schultz, Solid State Commun. 68, 437 (1988), where submicron segments of a ferromagnetic material were studied by self-induced anomalous Hall effect inside them.

${ }^{6}$ See, e.g., C. Beeli, B. Doubin, and P. Stadelmann, Phys. Rev. Lett. 75, 4630 (1995)

${ }^{7}$ Yu.V. Nazarov (private communication).

${ }^{8}$ X. Q. Li and F. M. Peeters, Superlattices Microstruct. 22, 2 (1997). 